\title{
Clinical Characteristics and Short-term Outcomes of Acute Low Frequency Sensorineural Hearing Loss With Vertigo
}

\author{
Myung Jin Park ${ }^{1, \star}$. Sang Hoon Kim" ${ }^{1, \star}$ Sung Su Kim² . Seung Geun Yeo ${ }^{1}$ \\ ${ }^{1}$ Department of Otorhinolaryngology-Head and Neck Surgery, Kyung Hee University School of Medicine, Seoul; ${ }^{2}$ Department of Biochemistry \\ and Molecular Biology, Medical Science and Engineering Research Center for Bioreaction to Reactive Oxygen Species, BK-21, Kyung Hee \\ University School of Medicine, Seoul, Korea
}

Objectives. This study analyzed short-term prognosis in patients with acute low frequency hearing loss (ALHL), and also investigate hearing recovery rates in patients with ALHL accompanied vertigo.

Methods. Retrospective medical record review of the patients who received treatment for ALHL between June 2005 and June 2015 were analyzed. Of the 84 patients, 53 were without vertigo, and 31 were with vertigo. Of the 31 patients, eight were treated with steroids, seven with diuretics alone, and 16 with both. Clinical and auditory characteristics before and after treatment were compared in these three groups.

Results. Pure tone audiometry after 8 weeks of treatment showed that patients with vertigo had significantly higher than patients without vertigo $(P=0.020)$. Patients with vertigo who recovered from ALHL had a greater tendency to receive early treatment than patients who did not recover. Patients who received the two steroid therapy groups (steroids alone and steroids plus diuretics) had a higher recovery rate than patients who received diuretics alone $(P=0.043$ and $P=0.037$, respectively).

Conclusion. The prognosis of patients with ALHL is worse in those with vertigo compared to without vertigo. The hearing recovery rate in patients with vertigo tends to be higher in those treated with steroids than with diuretics alone.

Keywords. Low Frequency Hearing Loss; Vertigo; Steroids; Diuretics

\section{INTRODUCTION}

Acute low frequency hearing loss (ALHL) was first described in 1981 as a sensorineural hearing loss confined to low frequencies and accompanied by tinnitus, autophonia and/or ear fullness [1]. The pathophysiologic mechanisms of ALHL are similar to those of sudden hearing loss, and the condition has been associated with cochlear hydrops and early stages of Meniere's disease [2].

\footnotetext{
- Received July 20, 2016

Revised October 17, 2016

Accepted October 31, 2016

- Corresponding author: Seung GeunYeo

Department of Otorhinolaryngology-Head and Neck Surgery, Kyung Hee University School of Medicine, 23 Kyungheedae-ro, Dongdaemun-gu, Seoul 02447, Korea

Tel: +82-2-958-8474, Fax: $+82-2-958-8470$

E-mail: yeo2park@gmail.com

*The first two authors contributed equally to this study.
}

Most patients with ALHL complain of hearing change, ear fullness or tinnitus rather than of hearing loss, and the short-term prognosis of ALHL has been reported to be better than that of sudden sensorineural hearing loss (SSHL). However, despite the clinical significance of progression of ALHL to Meniere's disease and of the recurrence of hearing loss, their pathophysiologic mechanisms remain unclear [3,4]. One of the main symptoms of Meniere's disease is episodic vertigo, accompanied by low frequency hearing loss. Alternatively, early stages of Meniere's disease may be characterized by hearing loss followed by vertigo, with clinical characteristics similar to those of ALHL, making their differential diagnosis difficult [5].

Despite numerous investigations of the pathophysiology and treatment of ALHL, there are to date no established diagnostic criteria or treatment guidelines for this condition. Most patients with ALHL without vertigo are treated with oral or intratympanic steroids [6,7], although some may be treated with diuret-

Copyright (C) 2018 by Korean Society of Otorhinolaryngology-Head and Neck Surgery.

This is an open-access article distributed under the terms of the Creative Commons Attribution Non-Commercial License (http://creativecommons.org/licenses/by-nc/4.0)

which permits unrestricted non-commercial use, distribution, and reproduction in any medium, provided the original work is properly cited. 
ics, either alone or with oral steroids [8-10]. The optimal initial treatment for ALHL patients with vertigo has not yet been determined, nor has whether this condition should be differentiated therapeutically from Meniere's disease. Moreover, it is unclear whether the co-occurrence of vertigo has an effect on the prognosis of patients with ALHL.

The objective of this study, therefore, was to determine the appropriate treatment for ALHL with vertigo, by comparing outcomes of different treatment methods, and by analyzing factors affecting clinical characteristics, hearing status and hearing recovery. And we assessed differences in the prognoses of ALHL patients with and without vertigo.

\section{MATERIALS AND METHODS}

We conducted this study in compliance with the principles of the Declaration of Helsinki. The protocol of this study was reviewed and approved by the Institutional Review Board of Kyung Hee University Hospital (IRB No. 2017-01-009). Written informed consents were obtained.

The medical records of 84 patients aged $\geq 18$ years who complained of tinnitus, ear fullness and/or hearing loss, but had normal tympanic membranes, and who received treatment for hearing loss confined to low frequencies on pure tone audiometry (PTA) between June 2005 and June 2015 were retrospectively analyzed. Patients were diagnosed with ALHL if they had otologic symptoms; average unilateral sensorineural hearing loss $>30 \mathrm{~dB}$ at two connected low frequencies, 250 and $500 \mathrm{~Hz}$, based on PTA; and normal hearing levels $<25 \mathrm{~dB}$ at hearing thresholds of 1, 2, 3, 4, and $8 \mathrm{kHz}$ [8]. Patients were excluded if they had Meniere's disease, acute or chronic otitis media, autoimmunologic ear disease, labyrinthitis fistula, or history of hearing loss or vertigo, or who met the audiological criteria for SSHL (particularly if accompanied by hearing loss of $1 \mathrm{kHz}$ ). The duration and frequency of vertigo, defined as subjective regardless of nystagmus, and of otologic symptoms, such as hearing loss, tinnitus and ear fullness, were analyzed.

Patients were treated with oral prednisolone, starting at 60 $\mathrm{mg} /$ day on days $1-4,40 \mathrm{mg} /$ day on days 5 and $6,20 \mathrm{mg} /$ day on days 7 and 8 , and $10 \mathrm{mg} /$ day on days 9 and 10 . Patients who showed no sign of recovery within 2 weeks of treatment initiation, and those unable to take oral steroids for any reason, received intratympanic steroid injections twice weekly for 2

\section{H}

- The prognosis in patients with acute low frequency hearing loss is poorer in those with than without vertigo.

- The hearing recovery rate in patients with vertigo tends to be higher in those treated with steroids than with diuretics alone. weeks. Some patients were treated with a diuretic, consisting of $25 \mathrm{mg}$ hydrochlorothiazide once daily for 14 days or longer. Of the patients in the ALHL group without vertigo, 29 were treated with oral steroids alone, 15 with oral plus salvage steroids, and nine with steroid injections alone. Of the patients in the ALHL group with vertigo, four were treated with oral steroids, four with oral plus salvage steroids, 10 with oral steroids and diuretics, six with oral plus salvage steroids plus diuretics, and seven with diuretics alone.

Hearing thresholds were measured at 250 and $500 \mathrm{~Hz}$ by PTA before and after 8 weeks of treatment. The degree of audiological changes was classified into five grades [8]: complete recovery, defined as a reduction in hearing thresholds to $\leq 25 \mathrm{~dB}$, which is within normal range, at all frequencies below $500 \mathrm{~Hz}$; partial recovery, defined as a $\geq 10 \mathrm{~dB}$ reduction, relative to baseline, in hearing thresholds at frequencies below $500 \mathrm{~Hz}$; unchanged, defined as $<10 \mathrm{~dB}$ differences in hearing thresholds from before to after treatment; progression, defined as $>10 \mathrm{~dB}$ increases in hearing thresholds at all low frequencies $<500 \mathrm{~Hz}$; and fluctuation, defined as a recurrence of $\geq 30 \mathrm{~dB}$ reduction in hearing function at low frequency after complete or partial recovery. Based on these definitions, patients were divided into a recovery group, which included patients with complete and partial recovery; and a non-recovery group, which included patients with unchanged, progressive and fluctuated hearing loss. Puretone hearing threshold was calculated as one fourth the sum of hearing at $500 \mathrm{~Hz}, 1 \mathrm{kHz}, 2 \mathrm{kHz}$, and $4 \mathrm{kHz}$; and mean low tone hearing threshold was calculated as the average hearing thresholds at 250 and $500 \mathrm{~Hz}$.

\section{Statistical analysis}

Audiograms obtained before and after treatment, as well as clinical characteristics of patients with and without vertigo, were compared by chi-square tests and $t$-tests. The Mann-Whitney $U$ test was used to compare age, sex, symptoms, time before treatment and hearing thresholds among the three treatment groups, as well as between the recovery and non-recovery groups. Hearing thresholds before and after treatment were compared by the Wilcoxon signed-rank test, and recovery rates of the three treatment groups were compared using the Kruskal-Wallis test. All statistical analyses were performed using the IBM SPSS ver. 21.0 (IBM Corp., Armonk, NY, USA), with a $P$-value $<0.05$ regarded as statistically significant.

\section{RESULTS}

Of the 84 patients, 53 (63\%) were diagnosed with ALHL without vertigo. Before treatment, the mean hearing threshold on the affected side was $27.5 \mathrm{~dB}(10-42.5)$ and the mean threshold at low frequencies was $50 \mathrm{~dB}$ (27.5-70); after treatment, these thresholds were $13.7 \mathrm{~dB}(3.7-40)$ and $18 \mathrm{~dB}(2-57.5)$, respec- 
Table 1. Clinical characteristics of patients with ALHL with and without vertigo

\begin{tabular}{|c|c|c|c|}
\hline Variable & ALHL without vertigo $(n=53)$ & ALHL with vertigo $(n=31)$ & $P$-value \\
\hline Age (yr) & $39(22-64)$ & $46(18-68)$ & 0.584 \\
\hline Sex (male:female) & $13: 40$ & $14: 17$ & 0.103 \\
\hline Side (right:left) & $20: 33$ & $9: 22$ & 0.698 \\
\hline Onset of treatment (day) & $5(1-20)$ & $7(1-21)$ & 0.130 \\
\hline \multicolumn{4}{|l|}{ Underlying disease } \\
\hline Diabetes & $3(6)$ & $2(6)$ & 0.121 \\
\hline Hypertension & $4(7)$ & $4(13)$ & 0.351 \\
\hline \multicolumn{4}{|l|}{ Accompanying symptom } \\
\hline Tinnitus & $42(82)$ & $30(97)$ & $0.044^{*}$ \\
\hline Ear fullness & $39(74)$ & $26(84)$ & 0.131 \\
\hline Initial hearing level (dB) & $27.5(10-42.5)$ & $28.7(11.2-42.5)$ & 0.092 \\
\hline Initial low tone hearing level $(\mathrm{dB})$ & $50(27.5-70)$ & $45(25-70)$ & 0.234 \\
\hline Initial opposite hearing level (dB) & $8.7(2-18)$ & $12.5(5-26.5)$ & 0.731 \\
\hline Final hearing level (dB) & $13.7(3.7-40)$ & $23.7(11.2-56.2)$ & 0.079 \\
\hline Final low tone hearing level (dB) & $18(2-57.5)$ & $40(5-72.5)$ & $0.020^{*}$ \\
\hline
\end{tabular}

Values are presented as median (range) or number (\%).

ALHL, acute low frequency hearing loss.

${ }^{*} P<0.05$.

Table 2. Clinical factors associated with hearing recovery in patients with ALHL accompanied vertigo

\begin{tabular}{lccc}
\hline Variable & Recovery group $(\mathrm{n}=21)$ & Non-recovery group $(\mathrm{n}=10)$ & $P$-value \\
\hline Age (yr) & $46(18-63)$ & $43(20-68)$ & 0.231 \\
Sex (male:female) & $9: 12$ & $5: 5$ & 0.551 \\
Side (right:left) & $6: 15$ & $3: 7$ & 0.701 \\
Onset of treatment (day) & $7(1-13)$ & $10(2-21)$ & $0.044^{*}$ \\
Underlying disease & & & 0.143 \\
$\quad$ Diabetes & $1(5)$ & $1(10)$ & 0.152 \\
$\quad$ Hypertension & $2(9)$ & $2(20)$ & 0.254 \\
Accompanying symptom & $20(95)$ & $9(90)$ & 0.441 \\
$\quad$ Tinnitus & $19(90)$ & $7(70)$ & 0.143 \\
$\quad$ Ear fullness & $27.5(11.2-41.2)$ & $27.5(13.7-42.5)$ & 0.151 \\
Initial hearing level (dB) & $47(25-62.5)$ & $42.5(35-70)$ & $0.001^{*}$ \\
Initial low tone hearing level (dB) & $27(16.2-56.2)$ & $0.001^{*}$ \\
Final hearing level (dB) & $22.5(11.2-43.5)$ & $42.5(35-72.5)$ & 0.693 \\
Final low tone hearing level $(\mathrm{dB})$ & $37.5(5-65)$ & & 0.452 \\
Treatment modality & & $2(20)$ & 0.174 \\
$\quad$ Steroid & $12(57)$ & $4(40)$ & $4(40)$ \\
$\quad$ Steroid+diuretics & $3(14)$ & & \\
Diuretics & & & \\
\hline
\end{tabular}

Values are presented as median (range) or number (\%).

ALHL, acute low frequency hearing loss; Recovery group, complete recovery+partial recovery; Non-recovery group, unchanged+progression+fluctuation. ${ }^{\star} P<0.05$.

tively (Table 1).

The remaining 31 patients (37\%) were diagnosed with ALHL accompanied vertigo. Before treatment, the mean hearing threshold on the affected side was $28.7 \mathrm{~dB}(11.2-42.5)$ and the mean threshold at low frequencies was $45 \mathrm{~dB}$ (25-70); after treatment, these thresholds were $23.7 \mathrm{~dB}(11.2-56.2)$ and $40 \mathrm{~dB}$ (5-72.5), respectively (Table 1 ).

A comparison of the two groups of ALHL patients with and without vertigo showed no differences in age, sex, affected side, onset of treatment, percentage with ear fullness, presence of underlying diseases and mean hearing thresholds on the affected side. However, tinnitus was significantly more frequent in patients with than without vertigo $(P=0.044)$, and the mean low tone hearing threshold after treatment was significantly higher in patients with than without vertigo $(P=0.020)$ (Table 1$)$.

Factors influencing hearing recovery of ALHL patients with vertigo were evaluated by comparing those who experienced recovery (including complete and partial recovery) and non-re- 


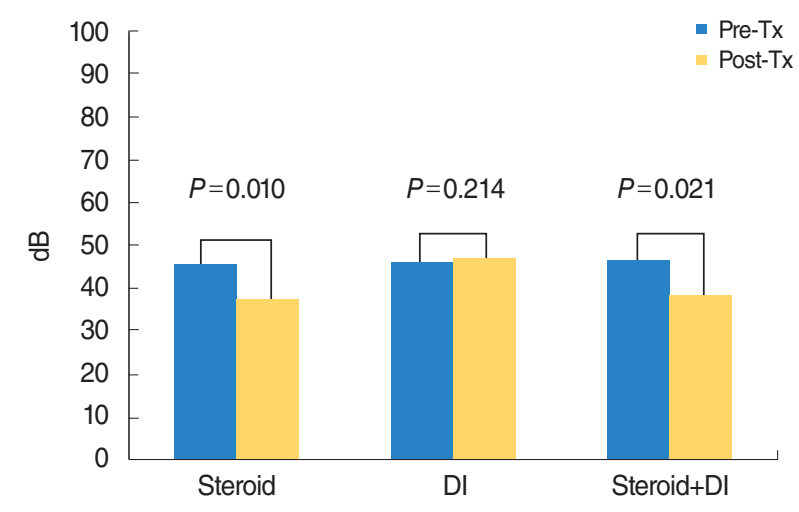

Fig. 1. Low tone hearing levels in patients with acute low frequency hearing loss and vertigo before and after 8 weeks of treatment. Tx, treatment; DI, diuretics.

covery (including unchanged, progression and fluctuation) after treatment, as assessed by PTA. The time between symptom onset and treatment initiation was significantly longer in the nonrecovery group $(P=0.044)$ (Table 2$)$. However, there were no between-group differences in age, sex, affected side, presence of tinnitus, ear fullness, audiograms of the affected side before treatment and underlying disease (Table 2).

Treatment outcomes in patients diagnosed with ALHL with vertigo were analyzed by comparing mean low tone hearing thresholds in three groups of patients: eight treated with oral steroids, with or without intratympanic steroids; seven treated with diuretics alone; and 16 treated with combinations of steroids and diuretics. Patients treated with steroids showed a statistically significant change in PTA, from $45 \mathrm{~dB}(35-60)$ before treatment to $37.5 \mathrm{~dB}(10-47.5)$ after treatment $(P=0.010)$. Similarly, patients treated with combinations of steroids and diuretics showed a statistically significant change in PTA, from $47.5 \mathrm{~dB}$ (25-65) before treatment to $37.5 \mathrm{~dB}(5-65)$ after treatment $(P=$ $0.021)$. However, patients treated with diuretics alone showed no change in low frequency PTA, from $45 \mathrm{~dB}(32.5-65)$ before treatment to $50 \mathrm{~dB}(27.5-72.5)$ after treatment $(P=0.214)$ (Fig. 1).

Of the eight patients treated with steroids alone, four $(50 \%)$ recovered completely, three (38\%) recovered partially, one $(12 \%)$ was unchanged, and none showed progression (Fig. 2). The complete recovery rates in the steroids alone and steroids plus diuretics groups did not differ significantly $(P=0.083)$; however, the complete recovery rates were significantly higher in the two steroid therapy groups (steroids alone and steroids plus diuretics) than in the diuretic alone therapy group $(P=0.043$ and $P=0.037$, respectively) (Fig. 2).

\section{DISCUSSION}

SSHL is sensorineural hearing loss that occurs rapidly and inexplicably at three connected frequencies, $30 \mathrm{~dB}$ or higher, partic-

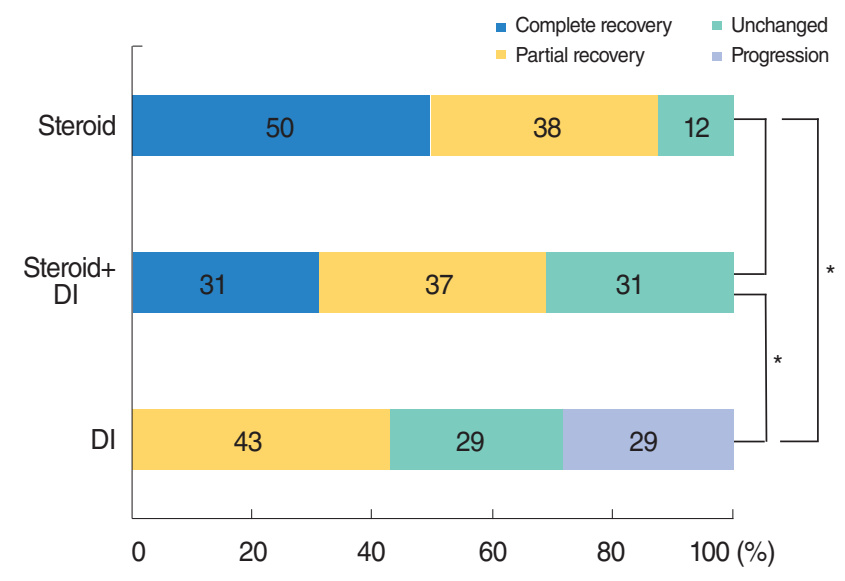

Fig. 2. Distribution of audiologic responses after 8 weeks of treatment in patients with acute low frequency hearing loss accompanied vertigo. DI, diuretics. *Significant difference between groups, $P<0.05$.

ularly at $1 \mathrm{kHz}$, within 3 days of onset [11]. Some patients experience hearing problems only at low frequency, a condition described as acute low tone hearing loss [8,12,13], ALHL [14], endolymphatic hydrops without vertigo, cochlear hydrops, or cochlear Meniere's disease [15].

Although audiologic criteria are necessary to accurately diagnose ALHL and to compare treatment outcomes, no universal definition has yet been established. ALHL has been defined as the sum of hearing thresholds at 2,4 , and $8 \mathrm{kHz}$ below $60 \mathrm{~dB}$ and the sum of hearing thresholds at 125,250 , and $500 \mathrm{~Hz}$ above $100 \mathrm{~dB}$ [12]. Alternatively, ALHL has been defined as the sum of hearing thresholds on the affected side at 125, 250, and $500 \mathrm{~Hz}$ above $80 \mathrm{~dB}$, the sum on hearing thresholds on the contralateral side more than $40 \mathrm{~dB}$ lower, and the difference between the two ears $10 \mathrm{~dB}$ at $1-8 \mathrm{kHz}$ being below $10 \mathrm{~dB}$ [9]. The subjects of the current study consisted of patients with ALHL who had degraded hearing acuity at 250 and $500 \mathrm{~Hz}$ and normal hearing acuity at 1, 2, 3, 4, and $8 \mathrm{kHz}$ [16]. In contrast, patients with degraded hearing acuity at $1 \mathrm{kHz}$ were excluded, as these audiologic criteria are considered diagnostic of SSHL. Additional studies are needed to determine diagnosis criteria for ALHL.

The major symptoms of ALHL include hearing loss, tinnitus, ear fullness and autophonia [12,17]. Patients with ALHL have a better prognosis than those with SSHL. The recurrence rate of ALHL has been reported to range from $9 \%$ to $47 \%$, with approximately $10 \%$ to $30 \%$ of these patients progressing to Meniere's disease [12,17]. It has been hypothesized that ALHL and Meniere's disease are different stages of the same type of immune endocrine disease [18], with several studies suggesting that ALHL is an early stage of endolymphatic hydrops confined to the cochlea. The study subjects consisted of patients with hearing loss at low frequencies of 250 and $500 \mathrm{~Hz}$, accompanied by early stage disequilibrium or definitive episodic vertigo, 
which gradually diminished and eventually disappeared. Although hearing status is similar in ALHL with vertigo and Meniere's disease, patients without recurrence of vertigo were not regarded as having definite Meniere's disease. Differentiating ALHL from Meniere's disease is required for appropriate treatment of the former. Criteria for "probable Meniere's disease" may include two or more episodes of vertigo or dizziness, each lasting 20 minutes to 24 hours, along with fluctuating aural symptoms (hearing, tinnitus or fullness) on the same side.

Factors reported to affect treatment response and prognosis in patients with SSHL include time from symptom onset to the start of treatment, occurrence of vertigo or tinnitus, type of audiogram and early stage hearing loss [5]. Moreover, recovery rates have been reported to be higher in patients with hearing loss in low to medium frequencies than in patients with high frequency hearing loss or hearing loss across all frequencies. In particular, SSHL patients with vertigo tend to have poorer prognosis than those without vertigo, with earlier treatment initiation in the former leading to better prognosis [19]. Consistent with this finding, our study showed that prognosis was poorer in ALHL patients with than without vertigo, but was better in patients who started treatment during earlier than later stages. Although these findings suggest that the clinical characteristics of ALHL are similar to those of SSHL, they do not have the same pathological mechanisms of action or belong to the same category of disease. Rather, as previously demonstrated, diuretics have been effective in treating ALHL, suggesting that the two diseases likely belong to different categories. ALHL with vertigo is more likely to progress to another condition than ALHL without vertigo. However, the presence of vertigo does not mean that the two diseases are different. Etiologically, ALHL involves an autoimmune response of the endolymphatic sac, which induces endolymphatic hydrops $[8,19]$, suggesting that ALHL may be an early stage of endolymphatic hydrops. Thus, early stage ALHL may not be accompanied by vertigo, whereas ALHL with vertigo tends to have a higher probability of long-term progression to Meniere's disease.

Initial treatments for SSHL include oral steroids and/or intratympanic steroid injections, with the combination showing greater or equal improvement compared with either alone. Steroids are also the treatment of choice for ALHL, as this condition is characterized by an imbalance in Th1/Th2 lymphocytes, with steroids helping to counteract this imbalance [18]. In contrast, the ability of diuretics to improve hearing thresholds in patients with ALHL is unclear. Although diuretics have been reported to affect vertigo, by improving endolymphatic hydrops in patients with Meniere's disease, the audiologic benefits of diuretics in this condition have not been determined [20]. The combination of steroids and isosorbide has been reported to be more effective than either alone in restoring hearing in patients with ALHL [8], with diuretics alone not significantly influencing ALHL recovery [9]. Consistent with these results, our study found that treatment with steroids or steroids plus diuretics resulted in statistically significant audiometric improvements, whereas diuretics alone did not. Moreover, complete recovery rates were higher in patients treated with steroids or combinations of steroids and diuretics than in patients treated with diuretics alone. The etiology of ALHL may include both endolymphatic hydrops and an autoimmune mechanism [8]. Thus, steroid therapy would be more effective than diuretics that reduce endolymphatic hydrops. Steroids have immunosuppressive and anti-inflammatory activities and may reverse autoimmune hearing loss caused by a sodium-potassium imbalance in endolymph. The lower rate of complete recovery in patients treated with diuretics alone may also be attributed to the low numbers of patients in the three treatment groups, as well as to the marked differences in these numbers, which may result in statistical errors.

In contrast to a previous study [8], which showed that the combination of steroids and diuretics was more effective in restoring hearing than either steroids or diuretics alone, this study found no statistically significant differences between steroids alone and combination of steroids and diuretics, although both steroids and the combination of steroids and diuretics were more effective than diuretics alone. This discrepancy may have been due to the inclusion in the present study of patients who received salvage steroid injections as well as oral steroids, whereas the previous study only included patients who were treated with oral steroids.

The findings of this study suggest that, as in patients with SSHL, steroids should be included in the first line therapy for hearing recovery in ALHL patients with vertigo, with combination therapy considered depending on the duration of vertigo and the presence of recurrence. This study had several limitations. First, although treatment outcomes were assessed 8 weeks after initial treatment, long-term follow-ups were not performed. Thus, long-term changes in treatment outcomes, rates of recurrence of hearing loss and of fluctuating otologic symptoms were not determined. Second, the short-term period of this study prevented a determination of whether ALHL with vertigo progressed to Meniere's disease. Finally, the small sample size precluded comparative analysis of factors associated with vertigo. In conclusion, hearing recovery rate in patients with ALHL accompanied vertigo tends to be higher in those treated with steroids than with diuretics alone. Prognosis was poorer in ALHL patients with than without vertigo.

\section{CONFLICT OF INTEREST}

No potential conflict of interest relevant to this article was reported. 


\section{ACKNOWLEDGMENTS}

This work was supported by the National Research Foundation of Korea (NRF) grant \& funded by the Korean Government (Ministry of Science, ICT and Future Planning) (No. 20110030072), Republic of Korea.

\section{REFERENCES}

1. Abe T. Acute sensorineural hearing loss in low tone frequencies. Otolaryngology (Tokyo). 1982;54:385-92.

2. Kwak MY, Yu KK, Kang YK, Yoon SW, Shim HJ, An YH. Intratympanic steroid and oral diuretics combination therapy for acute low frequency sensorineural hearing loss without vertigo. Korean J Otorhinolaryngol Head Neck. 2015 Jan;58(1):19-24.

3. Fuse T, Aoyagi M, Funakubo T, Sakakibara A, Yoshida S. Short-term outcome and prognosis of acute low-tone sensorineural hearing loss by administration of steroid. ORL J Otorhinolaryngol Relat Spec. 2002 Jan-Feb;64(1):6-10.

4. Chen CN, Young YH. Differentiating the cause of acute sensorineural hearing loss between Meniere's disease and sudden deafness. Acta Otolaryngol. 2006 Jan;126(1):25-31.

5. Bespalova IN, Van Camp G, Bom SJ, Brown DJ, Cryns K, DeWan AT, et al. Mutations in the Wolfram syndrome 1 gene (WFS1) are a common cause of low frequency sensorineural hearing loss. Hum Mol Genet. 2001 Oct;10(22):2501-8.

6. Imamura S, Nozawa I, Imamura M, Murakami Y. Clinical observations on acute low-tone sensorineural hearing loss: survey and analysis of 137 patients. Ann Otol Rhinol Laryngol. 1997 Sep;106(9):74650.

7. Mattox DE, Simmons FB. Natural history of sudden sensorineural hearing loss. Ann Otol Rhinol Laryngol. 1977 Jul-Aug;86(4 Pt 1): 463-80.

8. Morita S, Suzuki M, lizuka K. A comparison of the short-term outcome in patients with acute low-tone sensorineural hearing loss.
ORL J Otorhinolaryngol Relat Spec. 2010 Oct;72(6):295-9.

9. Suzuki M, Otake R, Kashio A. Effect of corticosteroids or diuretics in low-tone sensorineural hearing loss. ORL J Otorhinolaryngol Relat Spec. 2006 Apr;68(3):170-6.

10. Stachler RJ, Chandrasekhar SS, Archer SM, Rosenfeld RM, Schwartz SR, Barrs DM, et al. Clinical practice guideline: sudden hearing loss. Otolaryngol Head Neck Surg. 2012 Mar;146(3 Suppl):S1-35.

11. Whitaker S. Idiopathic sudden hearing loss. Am J Otol. 1980 Jan; 1(3):180-3.

12. Yamasoba T, Kikuchi S, Sugasawa M, Yagi M, Harada T. Acute lowtone sensorineural hearing loss without vertigo. Arch Otolaryngol Head Neck Surg. 1994 May;120(5):532-5.

13. Wu CL, Young YH. Vestibular evoked myogenic potentials in acute low-tone sensorineural hearing loss. Laryngoscope. 2004 Dec;114(12): 2172-5.

14. Fushiki H, Junicho M, Aso S, Watanabe Y. Recurrence rate of idiopathic sudden low-tone sensorineural hearing loss without vertigo: a long-term follow-up study. Otol Neurotol. 2009 Apr;30(3):295-8.

15. Hillman TM, Arriaga MA, Chen DA. Intratympanic steroids: do they acutely improve hearing in cases of cochlear hydrops? Laryngoscope. 2003 Nov;113(11):1903-7.

16. Junicho M, Aso S, Fujisaka M, Watanabe Y. Prognosis of low-tone sudden deafness: does it inevitably progress to Meniere's disease? Acta Otolaryngol. 2008 Mar;128(3):304-8.

17. Oishi N, Inoue Y, Saito H, Kanzaki S, Kanzaki J, Ogawa K. Long-term prognosis of low-frequency hearing loss and predictive factors for the 10-year outcome. Otolaryngol Head Neck Surg. 2010 Apr;142(4): 565-9.

18. Luque AE, Orlando MS, Leong UC, Allen PD, Guido JJ, Yang H, et al. Hearing function in patients living with HIV/AIDS. Ear Hear. 2014 Nov-Dec;35(6):e282-90.

19. Fuse T, HayashiT, Oota N, Fukase S, Asano S, Kato T, et al. Immunological responses in acute low-tone sensorineural hearing loss and Meniere's disease. Acta Otolaryngol. 2003 Jan;123(1):26-31.

20. Greenberg SL, Nedzelski JM. Medical and noninvasive therapy for Meniere's disease. Otolaryngol Clin North Am. 2010 Oct;43(5):108190. 